\title{
Aprendizaje de las reglas del Fútbol a través del videojuego
}

Armenteros Gallardo, Manuel

Dpto. Periodismo y Comunicación Audiovisual.

Universidad Carlos III de Madrid

Facultad de Humanidades, Comunicación y Documentación

28903 Getafe, MADRID

\section{Resumen / abstract}

El siguiente artículo analiza las ventajas de los nuevos medios interactivos para facilitar el aprendizaje de las reglas del fútbol y la similitud en la estética desarrollada en los hipermedias educativos y los videojuegos, tomando como referencia el videojuego Pro Evolution Soccer 5 y la Guía Interactiva del Fuera de Juego, editada por FIFA.

Por otro lado, se analiza la estructura de estos materiales, la forma de presentar su contenido y la construcción gramatical utilizada. Asimismo, se examinan otros factores que influyen sobre el alto grado de aceptación que generan en el usuario.

Entre otras conclusiones, se confirma la utilidad de estos materiales multimedia interactivos para facilitar el aprendizaje, capaces de combinar el entretenimiento con la adquisición de conocimiento, convirtiéndoles en una herramienta muy útil en la formación deportiva.

The following article analyzes the advantages of the new interactive means to facilitate the learning of the rules of the football and the similarity in the aesthetics developed in you educational Hypermedia and the video games, taking the video game as a reference Pro Evolution Soccer 5 and the Interactive Guide of Law 11, edited by FIFA. On the other hand, there is analyzed the structure of these materials, the way of presenting the content and the grammatical used construction. Likewise, there are examined other factors that influence the high degree of acceptance that they generate in the user. Between other conclusions, confirms to itself the usefulness of these materials multimedia interactive to facilitate the learning, capable of combining the entertainment with the acquisition of knowledge, turning them into a very useful tool into the learning of sports.

Palabras clave:

Hipermedia, aprendizaje, videojuegos, interactividad y fuera de juego. 


\section{Aprendizaje a través del juego}

¿Se aprende con los videojuegos? Sí. La ingente realización de estudios concluyen confirmando la eficacia educativa de los hipermedias de entretenimiento en la formación. Su capacidad para embaucar, su grado de realismo y la dinámica del juego los podría convertir en auténticas herramientas para el estudio, lejos de los aburridos hipermedias educativos anteriores.

Sin embargo, por la idiosincrasia misma del medio, así como por el objetivo de los hipermedias de entretenimiento, el videojuego limita, filtra el acceso a unos determinados conocimientos y destrezas. Por el contrario, el hipermedia concebido como educativo puede ahondar en aspectos que el hipermedia deportivo no puede hacer porque perdería la dinámica del juego. Por otro lado, el hipermedia educativo está emulando ciertos aspectos que habían sido propios del hipermedia de entretenimiento y que aseguran una mayor aceptación del usuario. Estos son: grado de realismo, diseño, usabilidad y accesibilidad a los contenidos.

\section{Grado de realidad}

Al analizar las siguientes capturas pertenecientes a la Guía Interactiva del Fuera de Juego y al hipermedia Pro Evolution Soccer 5, se observa un alto grado de reproducción de la realidad visual de las representaciones.
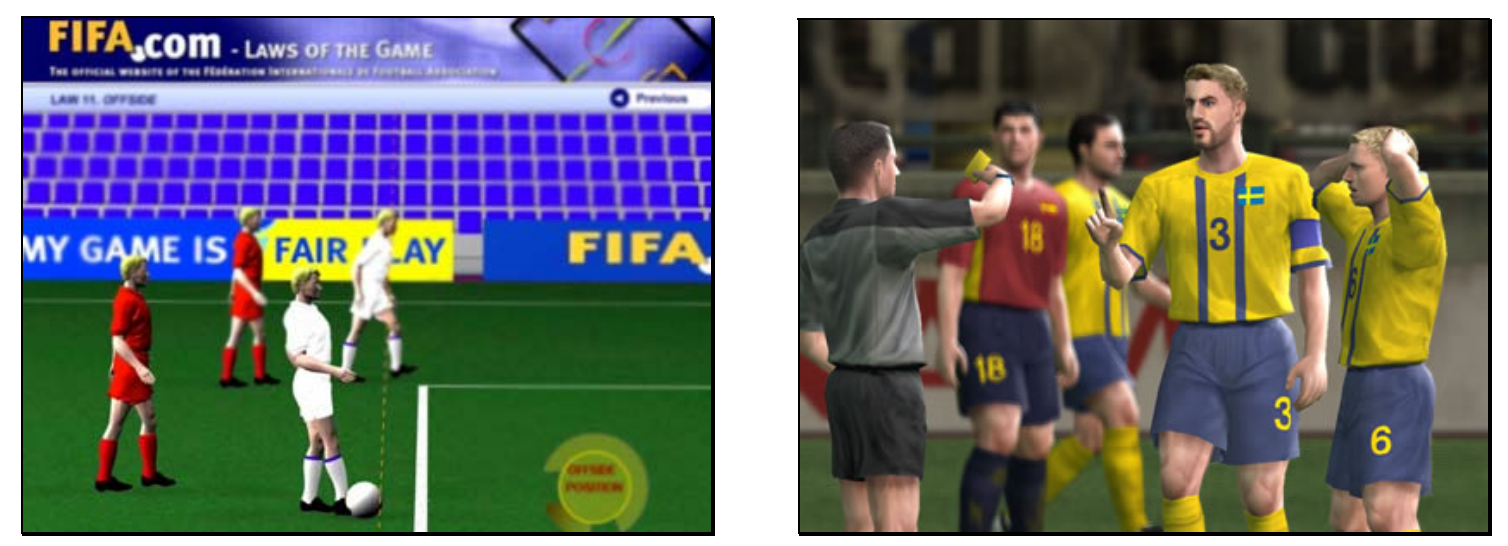

Ilustración 1. Captura de la guía interactiva del Fuera de Juego Ilustración 2. Captura del videojuego Pro Evolution Soccer 5

A mayor proximidad con la realidad visual representada, el usuario valora más positivamente el hipermedia ${ }^{1}$. La perspectiva, la profundidad de campo y las diferentes tonalidades han conseguido emular

\footnotetext{
${ }^{1}$ (Armenteros, 2005).
} 
las imágenes obtenidas por la lente de la cámara, dando como resultado un grado de realidad muy alto, que es uno de los medidores de calidad de un hipermedia.

Como puede observarse en los dos hipermedias analizados, la proximidad de la imagen visual a su referente es muy alta, no sólo en los parámetros de imagen estática como color, perspectiva, profundidad de campo, sino también en otros parámetros físicos como el movimiento de los jugadores.

Este salto tecnológico en la facilidad para reproducir la realidad visual recuerda el paso que dio la fotografía con respecto a la pintura, y el cine con respecto a la fotografía. Hoy día, la infografía 3d, permite reconstruir cualquier realidad visual basada en el mundo real o incluso no real, lugares y situaciones imaginables que no se corresponden con el mundo real. Es decir, es capaz de mostrar la realidad física y la virtual. Lo que existe ante nuestros ojos, y lo que no vemos, sino que imaginamos.

Sin embargo, la reproducción de la realidad visual está estrechamente ligada a cómo se muestra la realidad; qué se selecciona de esa realidad, qué interesa al usuario-espectador, y cómo accede este último a los contenidos. Cuando el realizador multimedia opta por un tipo de plano, un encuadre, una iluminación, un movimiento, etc. está mostrando un tipo de realidad visual, una forma de percibir las cosas que viene determinado por un conjunto de convenciones culturales.

El usuario responde a las convenciones del lenguaje audiovisual al cual está acostumbrado. Cuando comenzaron los trabajos de representación visual del guión de la Guía Interactiva del Fuera de Juego, pude comprobar cómo existe esa pregnancia del lenguaje audiovisual en los responsables de arbitraje de FIFA que mostraban una predilección hacia los encuadres que se utilizan en la realización de retransmisiones de fútbol en televisión. Encuadres y puntos de vista que también se han imitado en videojuegos y en otras representaciones hipermedias.

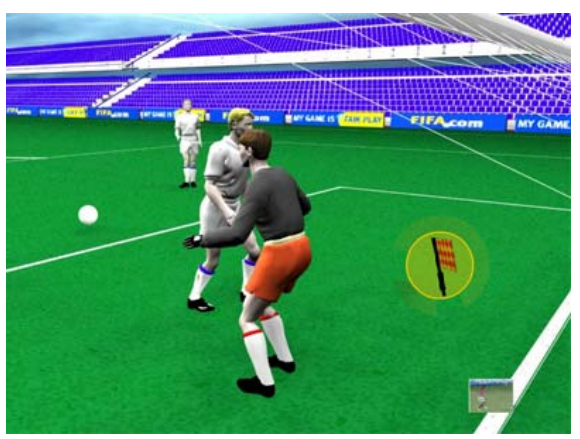

Ilustración 3. Captura de la Guía Interactiva del Fuera de Juego

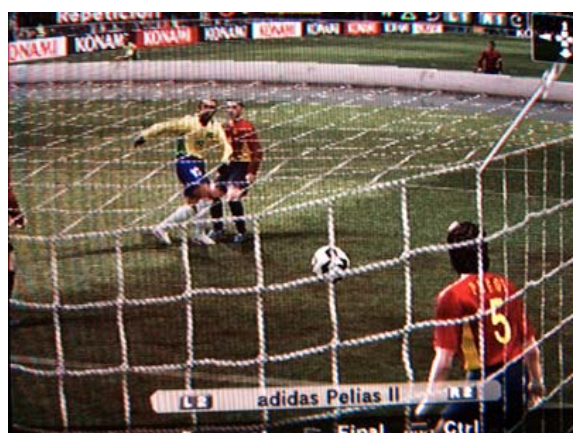

Ilustración 4. Captura del Pro Evolution Soccer 5 
Por tanto, la reconstrucción fidedigna de la realidad depende tanto de la percepción de la realidad del realizador como de las tipificaciones socialmente aceptadas de la realidad. De manera que la percepción de la realidad pasa por la capacidad para representar convenciones estéticas socialmente aceptadas, y por la capacidad para crear digitalmente un entorno o acción digital, así como por conocer las convenciones del lenguaje audiovisual que impregnan nuestra vida cotidiana. Por tanto, la reconstrucción de la realidad va a depender de cómo percibe el realizador la realidad, de las tipificaciones de la realidad socialmente aceptadas, de las convenciones estéticas del momento y de las convenciones del lenguaje audiovisual.

\section{La estética y la usabilidad}

La estética, la Ciencia que trata de la belleza y de la teoría fundamental y filosófica del arte (R.A.E.) es fundamental en la construcción formal de los hipermedias. Cada género hipermedia responde a unos criterios estéticos determinados. La cultura visual ha impregnado a todas las capas sociales de una idea de lo bello y de lo que no lo es. En este sentido, Manovich $(2006,241)$ afirma que la cultura visual de la era del ordenador es cinematográfica en su apariencia, digital en el plano material e informática en su lógica.

En la medida que la técnica avanzaba, las posibilidades expresivas tanto visuales como sonoras del medio eran mayores, hasta nuestros días, donde algunos videojuegos se han convertido en verdaderas obras de arte. Los creadores de videojuegos han explotado al máximo las posibilidades técnicas para crear atractivos hipermedias.

Los hipermedias educativos no pueden permanecer ajenos a esta evolución. Deben cuidar este aspecto, algo que no siempre se ha cumplido y que ha terminado asociando al hipermedia educativo con un recurso aburrido, relegado al aula, y al rincón de la estantería después de su primera instalación.

La Guía interactiva del Offside, en este sentido, ha apostado por un alto grado de realismo y por una estética, una convención establecida por FIFA en su libro de estilo, asegurando la aceptación del usuario en concordancia con el resto de publicaciones de la federación. 


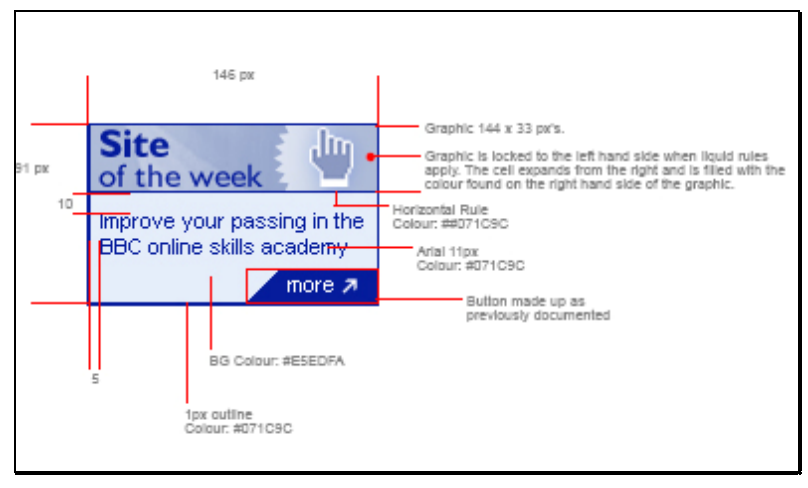

Ilustración 5. Captura del Libro de Estilo de FIFA

Por otro lado, la guía interactiva del Fuera de Juego ha respetado una interfaz similar a la interfaz desarrollada en la web. Puesto que la interfaz determina la experiencia del usuario sobre la obra, fue necesario establecer unos criterios de diseño, navegación y acción determinados, similares a los utilizados en la web de FIFA.com.

Es importante, pues, que el interfaz coincida con un conjunto de códigos reconocidos por el usuario. Por este motivo, desde las primeras reuniones en la sede de FIFA, atendiendo a uno de los objetivos establecidos el 27 de septiembre de 2004 por la Comisión de Árbitros y Árbitros Asistentes de la FIFA de crear un programa para garantizar un arbitraje de calidad universal en la Copa Mundial de la FIFA 2006, se empezó a definir las características del usuario a quien iría dirigido la guía interactiva, que cubriría tanto a árbitros y árbitros asistentes como al público en general.

Alcanzar un público heterogéneo no es algo fácil de resolver. Algo similar ocurre en los hipermedia deportivos, como el caso de Pro Evolution Soccer, que permite diferentes niveles de dificultad, pudiendo ser utilizado por jugadores de nivel avanzado como por jugadores con desconocimiento de las reglas y técnicas del fútbol.

Atender a diferentes usuarios exige contemplar el acceso a los contenidos desde diferentes grados de profundidad y detalle, de forma que el usuario con menos conocimientos pueda acceder a un primer nivel de información, mientras que los usuarios más avanzados puedan profundizar en aquellos aspectos o temas de su interés. De esta forma se evita que los usuarios menos cualificados abandonen el hipermedia por encontrarlo demasiado complejo, y al mismo tiempo se puede satisfacer la demanda de una información más elaborada para usuarios con un mayor grado de conocimiento como pueden ser los árbitros y árbitros asistentes. 
Por otro lado, la interfaz debe ser fácil de usar. La usabilidad contempla la facilidad con la cual el hipermedia permite al usuario alcanzar sus objetivos. Cada medio utiliza su propia interfaz, circunstancia que obliga al diseñador a conocer qué elementos del lenguaje son comunes entre las diferentes interfaces.

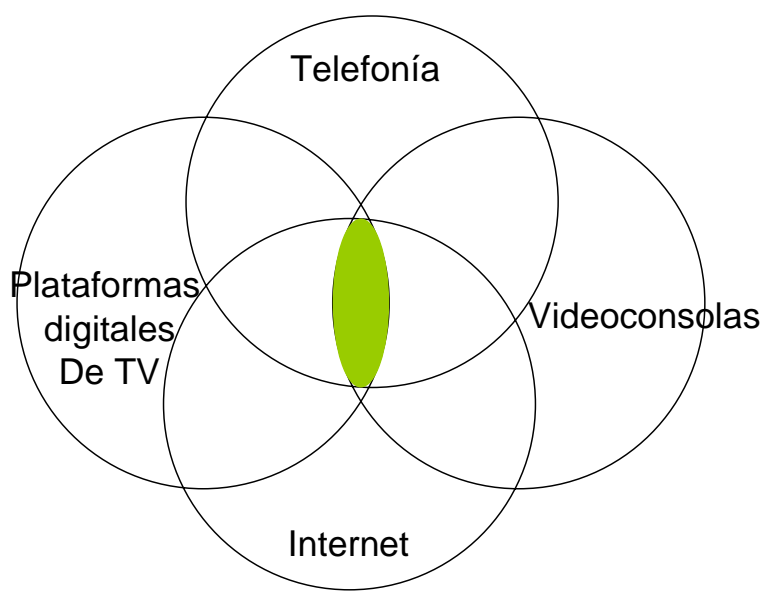

Ilustración 6. Zona común de convergencia entre interfaces

Una interfaz sencilla debe ser intuitiva y de fácil navegación. Si el usuario se siente obligado a leer la guía de uso antes de comenzar denota que existen códigos diferentes entre el usuario y la interfaz.

Cada soporte, no obstante, impone a la interfaz su propia idiosincrasia de navegación. En el caso de la Guía Interactiva del Fuera de Juego, la interfaz de acceso entre la aplicación hipermedia y el usuario se produce a través del teclado y del ratón, mientras que en el videojuego Pro Evolution Soccer 5 se lleva a cabo mediante el josting. La interfaz, en última instancia, va a determinar la forma en que el usuario concibe las relaciones con el hipermedia.
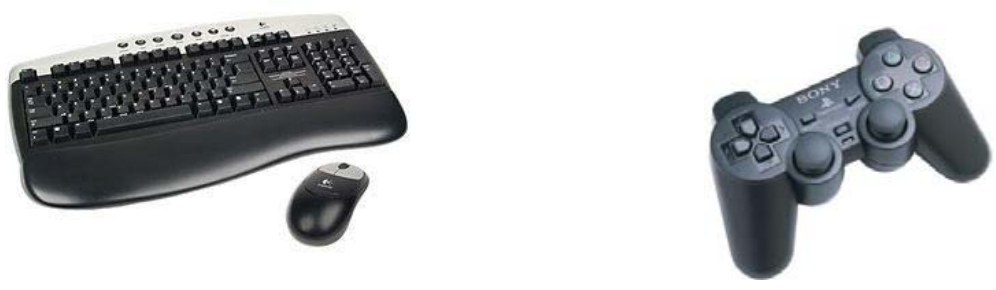

Ilustración 7. Interfaz de acceso para ordenador y videoconsolas

Cada tecnología ha generado unos hábitos de usabilidad diferentes. En ambos casos se requiere la utilización de las dos manos, pero en cada interfaz la asociación de elementos a las acciones que conectan están colocados espacial y temporalmente de forma diferente. Esta característica de los 
interfaces conlleva que la utilización de una u otra tecnología para de acceso al hipermedia exija una práctica de uso diferente.

Para aprovechar esta práctica de uso, la interfaz de navegación de la Guía Interactiva del Fuera de Juego ha emulado la interfaz de navegación utilizada en la página web de FIFA.com, en la sección de Reglas del Juego $^{2}$. Al mantener en las mismas coordenadas espaciales los enlaces de navegación, se evita distraer al usuario de la experiencia de la obra, concentrándose en el contenido.
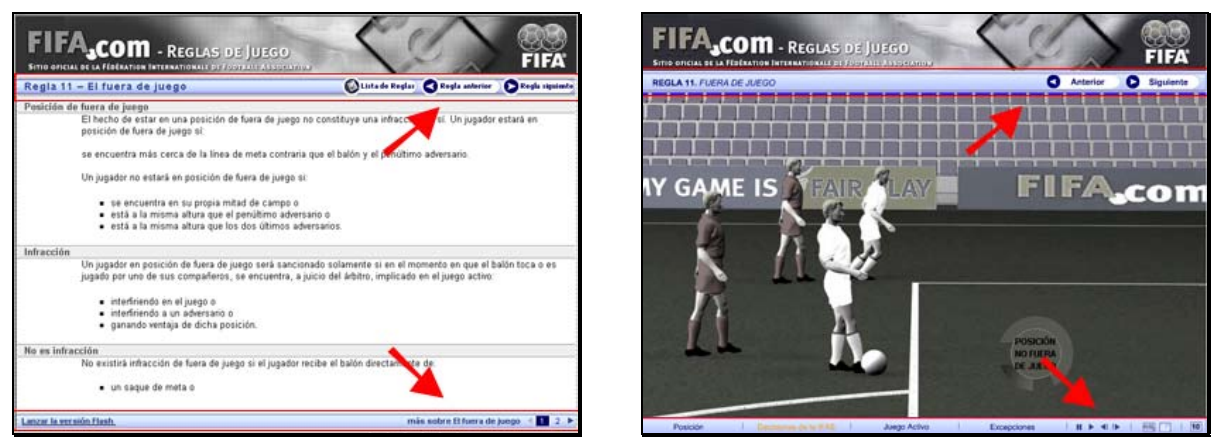

Ilustración 8. Captura de pantalla con las opciones de navegación en FIFA.com

Asimismo, la guía está diseñada para que discurra por sí sola, atendiendo en este aspecto a un discurso lineal. Está presentada en forma de pequeños objetos de aprendizaje, o diapositivas, que pasan de una a otra si el usuario no lo impide. Esta modalidad de navegación ha sido concebida como un híbrido entre el discurso lineal del DVD y el discurso hipertextual de Internet. Una lógica de navegación que responde a la necesidad de satisfacer una práctica de uso característica de otros soportes lineales como el vídeo y el DVD, y que son utilizados habitualmente en los materiales didácticos elaborados por FIFA.

En la medida que transgredimos una convención provocamos en el usuario un esfuerzo. Las convenciones, como las rutinas, permiten que el usuario pueda centrarse en el análisis de aquella información que necesita para desarrollar el conocimiento sobre algo. Si no existieran las rutinas no podríamos atender a una conversación telefónica y saludar con la mano al mismo tiempo; aún más si cabe, ¿se imagina el lector que cada vez que da un paso tuviéramos que pensar cuál es el pie que viene a continuación?

Para conseguir una navegación intuitiva el usuario tiene que tener los mandos de uso en el mismo lugar espacial durante toda la aplicación. Por ejemplo, todos los conductores de automóviles tienen el dispositivo para cambiar de marcha al lado derecho, excepto los ingleses, de ahí la confusión que habrá

\footnotetext{
${ }^{2}$ http://www.fifa.com/es/regulations/regulation/0,1584,3,00.html
} 
experimentado el lector si ha conducido un coche inglés. Se observa, una vez más, la necesidad en converger hacia interfaces que aprovechen las experiencias de los usuarios en otros medios, dando como resultado, si es el caso, de una lógica propia de navegación.

\section{Las guías de aprendizaje}

Para que el usuario pueda acceder a esa lógica de navegación propia se recurre a las guías de aprendizaje. Aunque los principios de usabilidad afirman que un buen diseño es aquel que tiene que ser intuitivo, de fácil uso, las guías se convierten en un instrumento muy útil para el aprendizaje de la gramática de funcionamiento de la aplicación.

Cada hipermedia las asocia a un tipo de aprendizaje. En el videojuego Pro Evolution Soccer 5, se ha observado que también son utilizadas como guías de entrenamiento. El usuario puede ensayar de forma individual o en equipo jugadas y lanzamientos, técnicas de ataque y de defensa, consiguiendo un avanzado sistema de aprendizaje para el conocimiento de técnicas y destrezas motoras y visuales. Este entrenamiento no solo permite mejorar la motricidad fina del usuario por el uso del mando en sus infinitas combinaciones, sino que dotará a los jugadores de mayor resistencia y calidad técnica.

Como su nombre indica, la guía conduce o enseña el camino hacia el aprendizaje. Son un instrumento de utilización sencilla que explica o refuerza aquellos conceptos relacionados con la lógica de aprendizaje del hipermedia. Pueden ejercer las funciones de guía los pequeños globos de información o las simulaciones como la que se muestra en la ilustración de la derecha, y que se corresponde a una simulación que muestra la función de cada uno de los iconos en los materiales multimedia utilizados para el aprendizaje.

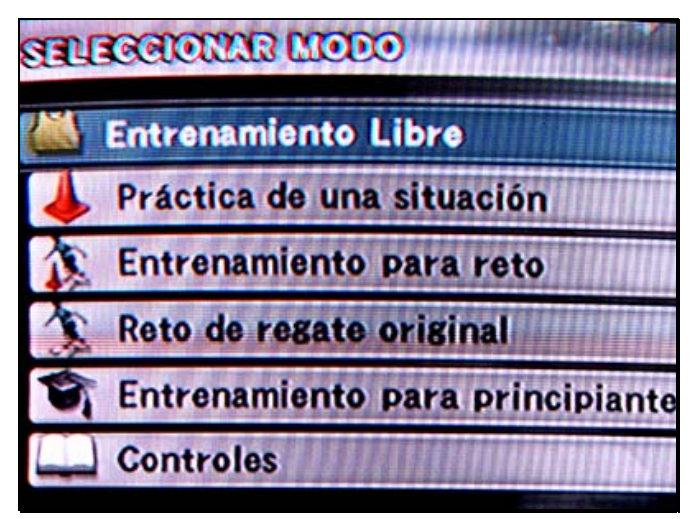

Ilustración 9. Simulación para ilustrar las funciones de los iconos utilizados en el interfaz de navegación.

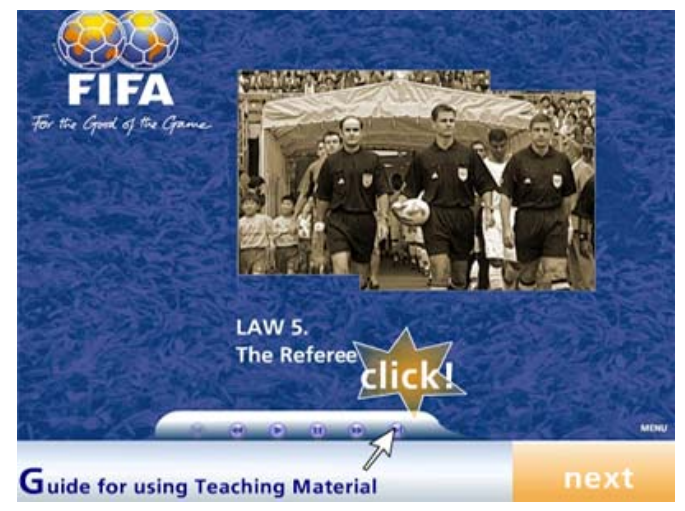

Guide for using Teaching Material 
Por otra parte, la elección de una correcta iconografía juega también un papel muy importante en una navegación de fácil uso. Actualmente ciertos iconos responden a un conjunto de convenciones establecidas y reconocidas por los usuarios. El icono es un signo que mantiene una relación de semejanza con el objeto o la acción que representa ${ }^{3}$. El icono, si es reconocido por el usuario, facilita la comunicación entre usuario y el hipermedia. Aunque no puede decirse que los iconos son universales, es cierto que funcionan para públicos muy diferentes.

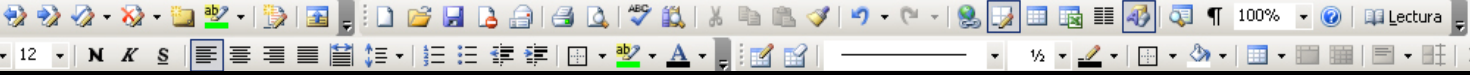

Ilustración 10. Paleta de herramientas totalmente iconográfica perteneciente al procesador de textos Office

Los primeros iconos aparecieron con el sistema operativo de los Macintosh. Con la expansión de Windows, las interfaces se han vuelto iconográficas debido a la semejanza que guardan con los objetos y acciones que representan, convirtiéndose en una forma de acceso a la información rápida e intuitiva.

\section{La accesibilidad a los contenidos}

La accesibilidad no es un término que se utilice exclusivamente para hablar del acceso de las personas con discapacidad, sino también de ofrecer al usuario unos contenidos a los cuales se pueda acceder sin obstáculos ni dificultades. Las barreras pueden venir dadas por la configuración de la pantalla, bien sea del monitor del ordenador, bien sea del televisor. Aunque cada vez existe más parecido entre las interfaces culturales ${ }^{4}$ y las interfaces de usuario, el escritorio del ordenador y la mesa de trabajo, el primero carece hoy por hoy de la tercera dimensión, de la profundidad. Por ejemplo, habrá observado el lector que muchas aplicaciones hipermedia que hacen uso de la superposición de pantallas, dando como resultado el solapamiento de ventanas y confundiendo al usuario, sensación de pérdida similar a la que se produce cuando tapamos una hoja con otra que se en la mesa de trabajo. Los usuarios de macintosh, por ejemplo, pueden observar cómo el interfaz de mac no maximiza las pantallas de las aplicaciones, de manera que es más difícil perder la referencia del plano que queda debajo. El éxito original de Apple se debió en parte a su interfaz de usuario, que era mucho más fácil de entender que los interfaces de comando que existían.

\footnotetext{
3 El autor norteamericano Charles Sanders Peirce hace un exhaustivo análisis de la relación entre el signo y el objeto que representa. Si esta relación es de tipo material, el signo es un icono. Por ejemplo, una fotografía o un mapa. Si la relación es de contigüidad, el signo es un índice o indicio. Por ejemplo, una huella o el humo. Finalmente, si la relación es convencional, el signo es un símbolo. Por ejemplo, la palabra mesa.

${ }^{4}$ El autor Lev Manovich utiliza el término interfaz cultural para describir una interfaz entre el hombre, el ordenador y la cultura. Es decir, las maneras en que los ordenadores presentan los datos culturales y nos permiten relacionarnos con ellos.
} 
Los hipermedias de entretenimiento sobre videoconsolas, como ocurre en el juego analizado, han descartado el solapamiento de ventanas en la navegación, y cuando se ven obligados a situar una pantalla sobre otra, lo hacen de tal manera que el usuario no pierde la referencia del documento que existe en el plano inferior.

La necesidad de simplicidad es relevante en toda navegación. Hay que buscar la sencillez, así como responder a las necesidades y gustos de los usuarios.

La usabilidad y el diseño funcionan de forma simbiótica. Una aplicación pensada exclusivamente en la usabilidad permitiría que los usuarios realizasen funciones de forma ágil y rápida, pero correría el riesgo de que los usuarios se sientan desconectados, sin implicación ni sentido de pertenencia.

\section{¿Qué otros factores determinan la implicación del usuario en el hipermedia?}

Como puede observarse en los videojuegos, tanto usabilidad como diseño se cuidan en extremo. Las compañías de videojuegos analizan el comportamiento de los usuarios y se cuantifica el grado de satisfacción producido en el jugador.

Uno de los factores que influyen sobre el grado de satisfacción en el usuario es el grado de autoestima que genera la aplicación. Maslow, en su análisis sobre las necesidades del ser humano, concluyó que cuando las tres necesidades básicas (fisiológica, seguridad y amor, cariño y pertenencia) están satisfechas, el ser humano busca la auto-estima y respeto de los demás. Es decir, en cada partida el sujeto busca un nuevo reto, que puede ser vencer al ordenador o videoconsola, alcanzar un determinado récord o bien superar a su compañero.

Ese afán de superación es fundamental en la construcción de los videojuegos. En ocasiones el objetivo no es ganar, sino superar ciertos niveles de experiencia que permiten al usuario alcanzar nuevos territorios, codearse con jugadores de alto nivel, o llegar a zonas VIPS (reservadas a personas relevantes), como ocurre en los juegos masivos multijugador (en inglés MMOG, Massively Multiplayer Online Games).

Por otro lado, siguiendo las teorías del conflicto, los videojuegos sitúan al sujeto en situaciones próximas a la vida real, que pueden ir desde una situación de conflicto puro (juego de suma cero) o de pura negociación (suma positiva).

En los hipermedias educativos, el factor de superación sobre la máquina o el compañero no es tan importante como la necesidad de conocer, de adquirir un conocimiento que nos permita ser mejor valorado, y, por tanto, de ganarse el respeto de los demás. 
Ambos enfoques obligan a una forma diferente de abordar el desarrollo de aplicaciones hipermedia. Centrémonos en la forma de tratar y enseñar la regla del Orsay e los hipermedias analizados.

Cuando el Pro Evolution Soccer 5 detecta una posición de fuera de juego, detiene la jugada cuando el atacante en posición de fuera de juego corre hacia el balón o manifiesta una intención clara de jugar. Inmediatamente, se muestra al usuario una simulación desde una cámara que muestra en un mismo encuadre el momento del pase del balón y la posición de fuera de juego del compañero que recibe y juega el balón; la propia dinámica del juego, como ocurre en los partidos reales, ofrece una repetición simulada, muy breve, que impide detenerse para analizar el porqué de la posición de fuera de juego. Este factor temporal podría justificar que no se contemplen otras casuísticas de infracciones de fuera de juego como "interferir en el juego" o "ganar ventaja de la situación".

La Guía Interactiva del Fuera de Juego, desde una estrategia didáctica diferente, puede ahondar en el tratamiento de la regla 11 planteando una exposición de la información escalonada de manera que el usuario que iniciara el recorrido de la guía interactiva desde el principio pueda ir adquiriendo los conocimientos necesarios para entender la información que se presenta más adelante.

Esta exposición didáctica programada de menor a mayor complejidad en la información presentada, ha hecho posible la gran aceptación de la Guía del Fuera de Juego. Así, está siendo utilizada como referencia en el análisis de la evolución del juego por periodistas, según el último artículo publicado en The Independent, por instructores y por jóvenes que se inician en el aprendizaje de las reglas del juego ${ }^{5}$.

\footnotetext{
${ }^{5}$ Para ver el análisis sobre la utilización de la Guía Interactiva del Fuera de Juego desde las diferentes perspectivas ver The Independent 16 de junio de 2006 en http://sport.independent.co.uk/football/internationals/article703173.ece

), Asociación del Fútbol Argentino, boletín $n^{\circ} 3805 ;$ y http://www.my-youth-soccer-guide.com/soccer-rules.html.

Otras referencias a la guía del fuera de juego pueden encontrarse en:

http://www.belfasttelegraph.co.uk/html/worldcup 2006/features/offside.html

http://www.chicagopublicradio.org/programs/worldview/series/worldcup facts.asp

http://blogs.foxsports.com/TheScout/Rules/32088

http://www.communigate.co.uk/york/yorkrefereesassociation/page19.phtml

http://www.dinosoupliotis.com/

http://es.wikipedia.org/wiki/Fuera de juego
} 


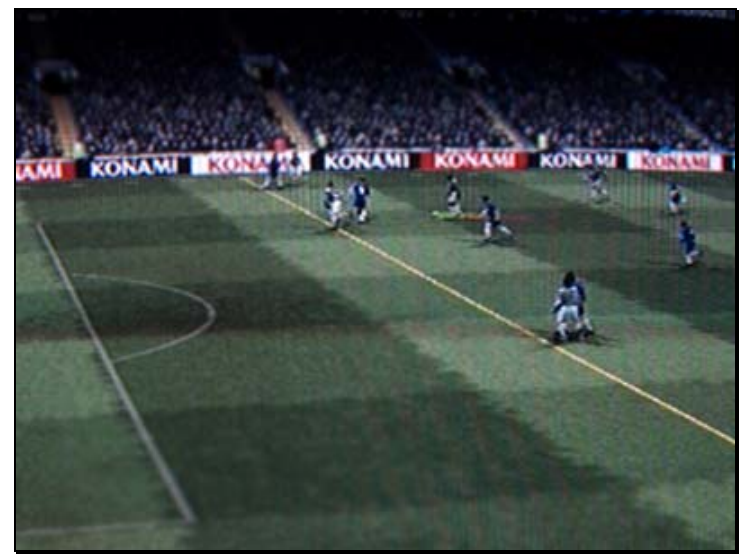

Ilustración 11. Captura del Pro Evolution Soccer 5 en un momento de fuera de juego

\section{Conclusiones:}

Existen diferencias de uso en los hipermedias de entretenimiento y los hipermedias educativos que implica estrategias didácticas diferentes.

La estética y la usabilidad son elementos formales muy valorados por los usuarios. Los hipermedias educativos no pueden descuidar la estética y la usabilidad, ni perder de vista que entre sus funciones han de responder a las necesidades del usuario. Asimismo, el grado de realismo de las representaciones visuales son un estímulo que genera un grado de aceptación alto en los usuarios.

Las guías de navegación y aprendizaje son un instrumento para conocer la propia lógica de la aplicación. Por otro lado, las guías de aprendizaje en los hipermedias deportivos pueden resultar un marco adecuado para profundizar en aquellos conocimientos que no pueden tratarse en mientras el usuario juega.

Finalmente, la interfaz, tanto de hardware como gráfica, determina la forma en que el usuario va a concebir las relaciones con la obra hipermedia.

Tanto los hipermedias deportivos como los hipermedias educativos pueden ser utilizados como herramientas para el aprendizaje del deporte. Su aplicación en los ámbitos de formación, no obstante, ha de ser contemplada de diferentes formas en función del medio y características del usuario. 


\section{BIBLIOGRAFÍA:}

Cabero, J. (2001). Diseño y utilización de los medios en la enseñanza. Barcelona: Paidos.

Manovich, Lev (2005). El lenguaje de los nuevos medios. Barcelona: Paidos.

Armenteros, M. (2005). Los espacios tridimensionales de los hipermedia educativos

navegados por agentes virtuales. Tesis Doctoral no publicada. Universidad Complutense, Facultad de Ciencias de la Información, España.

FIFA, (2005). Guía Interactiva del Fuera de Juego. Suiza: Federación Internacional de Fútbol, en http://www.fifa.com/es/regulations/regulation/0,1584,3,00.html 